\title{
El cine regional en México: en busca de una identidad neoleonesa
}

Regional Cinema in Mexico: In Search of a Nuevo Leon's Identity

\section{LuCila Hinojosa-Córdova Ihcordova@hotmail.com} https://orcid.org/0000-0003-0541-5347

Karen Jasso Vaquera karenjasso94@hotmail.com

Universidad Autónoma de Nuevo León, México.

FECHA DE RECEPCIÓN noviembre 11, 2019

FECHA DE APROBACIÓN mayo 15,2020

FECHA DE PUBLICACIÓN julio 1, 2020

https://doi.org/10.32870/ elojoquepiensa.v0i2 1.340
Resumen / El propósito de este trabajo es mirar a la cinematografía del tercer milenio realizada en el estado de Nuevo León, México, que como cine regional enfrenta las mismas dificultades estructurales que el resto del cine nacional, pero que por sus particularidades tiene ciertos rasgos que le confieren una identidad y valores culturales específicos. Como métodos se emplearon la investigación documental y la entrevista. Se presenta en primer lugar un panorama del cine mexicano actual, en cuyo contexto se desarrolla la cinematografía neoleonesa; en un segundo apartado se exponen algunos de los antecedentes del cine en la entidad y las etapas de su desarrollo; y en una tercera parte se presentan fragmentos de los testimonios de tres organizadores de eventos cinematográficos en la entidad y de tres cineastas comprometidos con el desarrollo del cine regional.

PALABRAS GLAVE / cine mexicano, cine regional, Nuevo León, identidad, valores culturales.
Abstract / The purpose of this paper is to take a look to the cinema of the third millennium in the Mexican state of Nuevo Leon, which as regional cinema, faces the same structural difficulties as the rest of the national cinema, but because of their peculiarities has certain features that give it an identity and specific cultural values. Documentary research and the interview were used as methods. First section presents a view of Mexican cinema today, the context in which the Nuevo Leon's cinematography develops; a second section sets out some of the history of cinema in the entity and the stages of its development; and a third section presents excerpts from testimonies of three film event organizers in the entity and three filmmakers committed to the development of regional cinema.

Keywords / Mexican cinema, regional cinema, Nuevo Leon, identity, cultural values. 
un $10^{\circ}$ lugar junto a otras entidades federativas, cuando años atrás había ocupado otras posiciones más importantes: en el 2016 estuvo en un tercer lugar; en 2017, en el cuarto; ya en 2018 decayó a un quinto lugar, compartido con otros estados.

La producción de largometrajes está centralizada en Ciudad de México. Según las cifras publicadas en los Anuarios estadísticos de cine mexicano 2017, 2018 y 2019, CDMX fue la entidad con mayor producción de cine nacional, con $36 \%$, $35 \%$ y $43 \%$ respectivamente. Sin embargo, cada vez se ve mayor participación de otras entidades; mientras que en 2015 la producción de películas cubría 68\% de los estados, en el 2017 abarcó alrededor de 90\% de la República mexicana.

No solo las montañas y la aridez del territorio norestense, sino paisajes de sus ciudades, sus avenidas, la música, la arquitectura, los coloridos grafitis, sus barrios y colonias con sus historias y personajes, son algunos de los elementos que le confieren identidad y están presentes en los trabajos de los cineastas que con sus proyectos cinematográficos buscan ocupar un lugar en la cinematografía mexicana e internacional proyectando nuestra identidad y valores culturales.

Este trabajo tiene el propósito de presentar un avance de una investigación más amplia acerca de la cinematografía que se produce en Nuevo León en el tercer milenio. Se presenta en primer lugar un panorama del cine mexicano actual en cuyo contexto se desarrolla la cinematografía neoleonesa; en un segundo apartado se exponen algunos de los antecedentes del cine en la entidad, para luego en un tercer lugar hablar de su situación actual con la descripción de la trayectoria de tres cineastas neoleoneses que se han distinguido por su producción de calidad y aportaciones a la cinematografía mexicana.

\section{PanORAMA DEL CINE MEXICANO ACTUAL}

La historia contemporánea del cine nacional está muy vinculada a la firma, en 1992, del Tratado de Libre Comercio de América del Norte (TLCAN, ahora T-MEC) entre México, Estados Unidos y Canadá. Las políticas económicas neo- liberales, que desde el régimen de Miguel de la Madrid se adoptaron en la década de 1980, con la consecuente apertura de los mercados, la aprobación de una nueva y no equilibrada Ley Federal de Cinematografía (decretada a las dos semanas de la firma del TLCAN), y la liberación del precio del boleto en taquilla, fueron algunos de los factores que dejaron desprotegida a esta industria que, aunado a factores externos derivados de la globalización económica, ocasionaron su desplome (ver Hinojosa, 2003, 2007a y 2019). De estos factores, el que más contribuyó a su crisis fue la nueva legislación de cine, ya que dejó desprotegida a la exhibición.

Luego de haberse firmado el TLCAN en 1992, siendo presidente Carlos Salinas de Gortari, se aprobó en el Congreso de la Unión una nueva Ley Federal de Cinematografía que abrogaba la vigente desde 1949, la cual se publicó en el Diario Oficial de la Federación el 29 de diciembre de 1992 (dos semanas después de la firma del TLCAN, que se firmó el 17 de diciembre). En la ley de 1949 se establecía que el 50\% de las pantallas de las salas de cine debían exhibir películas nacionales, que si bien no cubrían la cuota de pantalla ni satisfacían del todo las exigencias de los distintos públicos, estos asistían a verlas y la ley operaba como una especie de protección cultural; en la nueva ley, vigente desde 1992, se establece que a partir de 1993 las salas cinematográficas deberían exhibir películas nacionales en un porcentaje de sus funciones, por pantalla, no menor al $30 \%$ en $1993,25 \%$ en $1994,20 \%$ en $1995,15 \%$ en 1996, para quedar en un $10 \%$ en 1997 , y con una semana mínimo de exhibición.

Esta ley ha tenido varias reformas, pero no en lo sustancial y que tiene que ver con las restricciones a la exhibición. El año de 1997 fue el más crítico, con solo nueve películas producidas. ¿Quién iba a querer invertir en una película que solo se exhibiría en 10 de cada 100 pantallas?

Con todo y crisis, las películas Como agua para chocolate (Alfonso Arau, 1992) y Cilantro y perejil (Rafael Montero, 1998), comenzaron a llenar de nuevo las salas de cine en el país, atrayendo públicos nacionales e internacionales y 
recibiendo premios en festivales propios y extranjeros; la producción empezó a recuperarse lenta, pero promisoriamente, a partir de la segunda mitad de la década de 1990 y principios del 2000, cuando se empieza a vislumbrar un cambio paulatino en la oferta y consumo de cine mexicano con los éxitos de taquilla como Sexo, pudor y lágrimas (Antonio Serrano, 1999), Amores perros (Alejandro González Iñárritu, 2000), Y tu mamá también (Alfonso Cuarón, 2001) y la polémica El crimen del padre Amaro (Carlos Carrera, 2002), que hicieron pensar en una naciente recuperación de la industria cinematográfica nacional con los millones de dólares recaudados no solo en México, sino también en otros países como Estados Unidos de América, los premios y reconocimientos otorgados en festivales de cine a películas y cineastas mexicanos, además del regreso de los espectadores a las salas de cine, quienes parecen estar mostrando un gusto renovado por estas nuevas películas nacionales alcanzando un crecimiento sostenido a partir de 2006.

En el 2013, con los estrenos de No se aceptan devoluciones (Eugenio Derbez, 2013) y Nosotros los Nobles (Gary Alazraki, 2013), el porcentaje de la taquilla producto de la asistencia a ver cine mexicano fue de $10.9 \%$ del total, el porcentaje más alto en la última década. Ese año la asistencia a ver películas mexicanas fue de 30.1 millones de espectadores.

En los últimos años se ha visto un repunte de la presencia del cine nacional en cartelera y el regreso de los espectadores a las salas cinematográficas a ver su cine, lo que hace pensar en una "nueva Época de Oro del cine mexicano", aunado a las premiaciones nacionales e internacionales que han recibido algunos cineastas como Alejandro González Iñárritu, Guillermo del Toro y Alfonso Cuarón [TABLA 1].

Cabe señalar que el triunfo es de los cineastas, no del cine mexicano, ya que desafortunadamente, desde hace casi tres décadas, no existe una política cultural definida y operativa que impulse a esta industria audiovisual tan importante para la identidad y cultura nacional.
Algunos datos comparativos entre 1990 y 2019 ilustran lo anterior:

- En 1990, se produjeron 75 películas, de las cuales se estrenaron 74 en las salas de cine.

$\rightarrow$ En 2019, se produjeron 216, de las que se estrenaron solo 101.

- En 1990, México contaba con 1896 salas de cine comercial, es decir, el 25.3\% de la cantidad con las que contaba en el 2019 (7493), pero a las que todavía asistieron 197 millones de espectadores, cuando la población era entonces de 84 millones de habitantes de acuerdo a las estadísticas del Consejo Nacional de Población, lo que promediaba 2.3 de asistencia por habitante.

$\rightarrow$ En el 2019, con más población, 126.5 millones de habitantes, y casi un $75 \%$ más salas de cine, se contabilizaron 341 millones de espectadores, el promedio de asistencia por habitante fue de 2.7, ligeramente mayor al de 1990 .

$\rightarrow$ El precio del boleto en taquilla formaba parte de la canasta básica y era fijado y regulado por el gobierno federal hasta $1992^{1}$. Ese año el precio se liberó y ahora es fijado libremente por los distribuidores y exhibidores, aunque su regulación sigue siendo de carácter federal.

Con altas y bajas, el cine mexicano se recupera, pero no todo ha sido favorable en esta transformación. La exhibición de películas nacionales desafortunadamente sigue siendo la tarea pendiente, ya que no ha tenido los mismos incentivos

${ }^{1}$ Durante el sexenio de Carlos Salinas de Gortari (1988-1994) la inflación subió $521.12 \%$ de acuerdo con el Índice Nacional de Precios al Consumidor. Los productos que subieron más sus costos fueron la educación y el entretenimiento. El producto que aumentó más fue el boleto de cine: $491.5 \%$ (Hinojosa, 2003). 
TABLA 1. El cine mexicano en números.

\begin{tabular}{lcccccccc}
\hline & 2013 & 2014 & 2015 & 2016 & 2017 & 2018 & 2019 \\
$\begin{array}{l}\text { Pantallas } \\
\text { cinematográficas }\end{array}$ & 5547 & 5678 & 5977 & 6225 & 6633 & 7024 & 7493 \\
\hline $\begin{array}{l}\text { Películas extranjeras } \\
\text { estrenadas }\end{array}$ & 364 & 330 & 334 & 317 & 336 & 384 & 353 \\
\hline $\begin{array}{l}\text { Asistencia total* } \\
\text { Películas mexicanas }\end{array}$ & 101 & 68 & 80 & 90 & 88 & 115 & 101 \\
\hline estrenadas & 240 & 286 & 321 & 338 & 320 & 341 \\
\hline $\begin{array}{l}\text { Asistencia a ver } \\
\text { películas mexicanas* }\end{array}$ & 30.1 & 24 & 17.5 & 30.5 & 22.4 & 30.3 & 35.2 \\
\hline \begin{tabular}{l} 
Ingresos en taquilla** \\
\hline
\end{tabular} & 11860 & 11237 & 13334 & 14808 & 16142 & 16292 & 18659 \\
\hline
\end{tabular}

Fuente: Anuarios estadísticos de cine mexicano (IMCINE, 2014, 2015, 2016, 2017, 2018, 2019 y 2020).

(*) Millones de personas

(**) Millones de pesos 
que la producción. Si bien en los últimos años se ha incrementado el número de estrenos en pantalla, las cifras no llegan a la cantidad de estrenos que se tenían antes de los cambios en la legislación que se dieron paralelamente a la entrada del TLCAN, estrenos que eran más proporcionales a la producción, favoreciendo ahora más a las películas extranjeras, básicamente estadounidenses.

\section{Antecedentes DEL CINE EN NUEVO LEÓN}

Existen pocos registros del cine realizado en Nuevo León. El sitio en internet Filmografia mexicana (Filmoteca UNAM, s. f.), registra alrededor de 100 largometrajes filmados en Nuevo León, de 1907 a 2003, pero no todos realizados por cineastas locales. Desafortunadamente las fichas con las que se registra la filmografía no dan cuenta del origen de los cineastas ni se le dio continuidad al archivo.

Los Anuarios estadísticos de cine mexicano se empezaron a publicar hasta 2010. Aunque anterior a ello el Instituto Mexicano de Cinematografia (IMCINE) publicaba un informe anual, en estos no se documentaba la producción cinematográfica por entidad federativa, hasta hace cuatro años. Los Anuarios del IMCINE documentan como cine mexicano el realizado en el país, incluso coproducciones con otros países, y distingue como películas realizadas en las entidades las registradas como producidas en la entidad respectiva.

En la entidad existen pocos registros sobre el cine en Nuevo León. Sin embargo, se destacan dos trabajos: el de Diana González y Maximiliano Maza (2013), dos investigadores locales, quienes publicaron un libro donde documentan las fichas de la filmografía realizada en territorio neoleonés, desde sus primeros registros hasta 2013; y un esfuerzo más reciente, el de Kassandra Sifuentes Zúñiga (2019), quien publicó como libro su tesis de licenciatura en Historia sobre los inicios del cine en Monterrey. Con información de estas dos aportaciones y otros datos obtenidos de internet, se busca reconstruir los antecedentes del cine en la entidad.
El cine llegó a Nuevo León a finales del siglo XIX gracias al empeño de personajes de la localidad, entusiasmados por las modernas tecnologías de la época. Aunque existe una discrepancia respecto a la fecha exacta, se considera el 11 de marzo de 1898, poco más de un año después de su exitoso paso por la capital mexicana, el día que se llevó a cabo en Monterrey la primera función proyectada con un cinematógrafo de los hermanos Lumière, traído de Ciudad de México por el tipógrafo y empresario de espectáculos Lázaro Lozano (González y Maza, 2013; Sifuentes Zúñiga, 2019).

Esta primera exhibición se llevó a cabo en la galería e imprenta del fotógrafo Desiderio Lagrange, ubicada en la calle Hidalgo a unos metros de la plaza del mismo nombre. No se tiene el dato de cuáles fueron las primeras vistas exhibidas y la presentación se hizo entre amigos en algún pasillo del local, improvisando una sala de cine. Ese mismo año, los amigos de Lagrange y Lozano animaron a estos a instalar el cinematógrafo en un local más amplio, una pequeña sala improvisada en un salón de boliche, donde actualmente se ubica el Hotel Ancira, también sobre la calle Hidalgo a unos metros del local anterior, en el centro de Monterrey (Isidro Saldaña citado en Sifuentes Zúñiga, 2019, p. 50).

El primer registro de una película filmada en la entidad data de junio de 1907, cuando Carlos Mongrand - empresario de origen francés - realizó La Cervecería Cuauhtémoc de Monterrey como propaganda para esta naciente industria neolonesa que todavía es un ícono en la entidad (González y Maza, 2013; Sifuentes Zúñiga, 2019).

Las primeras películas realizadas en Nuevo León eran más bien documentales que registraban acontecimientos de la vida cotidiana en la ciudad: La llegada del general Álvaro Obregón a Monterrey, Nuevo León (Eustasio Montoya, 1915); La batalla de Villaldama, Nuevo León (Eustasio Montoya, 1915); Revista Monterrey (Ramón Marty, 1923); Exposición regional de Monterrey: Festejos patrios en Monterrey (José S. Ortiz, 1926), por mencionar algunas. 
Según los autores, en una primera etapa (1907-1926), la del cine silente, surgieron los pioneros del cine neoleonés como José S. Ortiz (ya citado), cineasta nacido en la entidad y que filmó también en otras ciudades del país, fotógrafo de profesión, considerado el primer cineasta nacido en Nuevo León. Su primera película como director y productor fue En la tierra del oro negro (1921), un documental acerca de un incendio de unos tanques de petróleo en la zona del sur de Tamaulipas y norte de Veracruz.

Un segundo periodo corresponde al periodo de 1940 a 1960, donde a pesar de contar con suficiente documentación y prestigio comprobado, ni las películas filmadas en el estado por cineastas nacionales, ni la obra fílmica de dos grandes realizadores nacidos en Nuevo León, como lo fueron Alejandro Galindo y Rogelio A. González, habían sido incluidas como parte de la historia de la cinematografía regional, la cual realizó hasta hace pocos años la Filmoteca Nacional. Curiosamente, tanto Galindo como González no desarrollaron su carrera en su región de origen, por lo que en sus filmes no encontramos rasgos estilísticos o temáticos específicos de la identidad neoleonesa.

Mientras estos dos cineastas desarrollaban sus producciones desde el centro del país, en Nuevo León se llevaron a cabo una serie de producciones industriales e incluso algunas las produjeron cineastas capitalinos, cuyas características y temáticas sí se identifican con lo neoleonés, por ejemplo, Manifestación

anticomunista en Monterrey (Gustavo Sáenz de Sicilia, 1936), que por la fecha de su realización puede considerarse como la primera película sonora filmada en Nuevo León de la que se tiene registro; Cuando lloran los valientes (Ismael Rodríguez, 1947), historia basada en la letra del corrido de Agapito Treviño Caballo Blanco; La ciudad de los niños (Gilberto Martínez Solares, 1957); Los pequeños gigantes (Hugo Butler, 1960), acerca de un equipo de béisbol formado en su mayoría por niños de familias muy humildes, quienes se convierten en los primeros no estadounidenses en obtener un campeonato de este deporte; $\boldsymbol{E l}$ pocho (1970), producida, dirigida y actuada por Eulalio González Piporro, en la que personifica a un mexicano-norteamericano que busca conciliar su doble ascendencia cultural.

Según González y Maza (2013, p. 145), en 1959 se fundó en Monterrey la primera productora cinematográfica local, Teen's Productions, de Gherardo Garza Fausti (1943-2007), productor, director y guionista de sus propias películas, y cuya filmografia es la más amplia de un cineasta contemporáneo neoleonés, quien realizó largometrajes, mediometrajes y cortos en diversos formatos. Uno de los rasgos característicos de sus obras es que la mayoría de sus películas se basan en adaptaciones de obras literarias. Le gustaba experimentar técnicamente con sus filmes, así como jugar con los planos y ritmos de montaje. Entre sus largometrajes podemos mencionar Selaginela (1967), Entre dos mundos (1968), Tiempo de encuentros (1972), y Obsesión de amor (1979).

El cine realizado entre 1960 y 1970 es considerado como la de los pioneros modernos del cine neoleonés. En esta etapa se produjeron documentales, dramas juveniles, adaptaciones literarias y trabajos de cine industrial, como las de Garza Fausti. A este periodo pertenecen también otros cineastas identificados con el cine experimental como Áliber Medrano, Roberto Escamilla y Víctor Saca. Es de notarse la variedad de formatos fílmicos utilizados por estos pioneros modernos: 8, 16 y $35 \mathrm{~mm}$, así como video de 2 pulgadas. Tal variedad permite vislumbrar la heterogeneidad característica del cine neoleonés en la producción posmoderna de Nuevo León.

Luego vino la época del denominado cabrito western (19811995), un cine cuyas particularidades de producción, temática y estilo le dieron este título sui generis (el cabrito asado es un platillo típico de la región noreste); la situación geográfica y ciertas condiciones apropiadas en materia de preparación técnica y experiencia humana dieron lugar al surgimiento de esta especie de subgénero en una época donde se reflejaban las situaciones y problemas de la población habitante de la zona limítrofe entre la región noreste y el estado de Texas, Estados Unidos. Nuevas generaciones surgidas de las 
universidades locales de las que egresaban profesionales de la comunicación interesados en hacer cine, así como una nueva fuerza de producción de largometrajes industriales realizados en la entidad, dio paso a este subgénero sin ambición alguna de calidad, pero que ya reflejaba en sus imágenes el emergente medio ambiente del contrabando, los indocumentados, los traficantes de drogas y las historias centradas en personajes norteños que se inmortalizaban a través de los corridos que narraban sus aventuras en las canciones. Fue en esta época que se desarrolló una producción cinematográfica importante con películas como Pistoleros famosos (José Loza, 1981), Cazador de asesinos (José Luis Urquieta, 1983), o El traficante (José Luis Urquieta, 1987). Entre los aspectos de identidad regional, las bandas sonoras de estas películas incluían casi siempre música de grupos norteños de mucho arraigo en el gusto del público, que llenaba las salas de exhibición de tal manera que ninguna otra producción regional posterior ha podido igualar.

La primera mitad de la década de 1990 fue muy crítica para el cine mexicano dadas las condiciones del contexto nacional surgido a partir de la entrada en vigor del TLCAN y, con ello, la de México a la globalización. Sin embargo, como se había mencionado, el público no abandonó del todo a su cine, y este volvió a tener presencia en las salas tanto nacionales como internacionales. Ejemplo de ello fue Como agua para chocolate (Alfonso Arau, 1992), que impuso una marca de permanencia en México y de distribución en salas de Estados Unidos. En Monterrey, la punta de lanza para la cinematografía regional la constituyó En el paraíso no existe el dolor (Víctor Saca, 1993), filmada en $35 \mathrm{~mm}$ en distintos escenarios de la zona metropolitana de Monterrey y financiada parcialmente por el gobierno del estado de Nuevo León, quien estableció una diferencia práctica con sus antecesores generacionales al producir esta película con criterios industriales, aunque no se estrenó hasta 1998. También, en 1994, Gherardo Garza Fausti estrenó en la ciudad Crónica de un amargo despertar, largometraje que no llegó a las salas comerciales debido a su formato en video y por la participación de jóvenes universitarios en lugar de actores profesionales.

Este empuje del cine neoleonés que inició en la década de 1990 y se prolongó al siglo XXI encontró resonancia en la prensa general de la región, reflejada en los encabezados que destacaron la "avalancha del cine regio" y la notable participación que algunas de sus producciones lograron en prestigiados festivales de cine como Sundance y Venecia. Gracias a esta tendencia, durante los primeros años del siglo XXI se presentó un aumento considerable en las producciones de cine regional, tanto de largometrajes como de cortometrajes, y fueron estos últimos, de hecho, superiores en número a los primeros, pero a la vez ha sido de ambos el mérito de instituir un nuevo movimiento dentro del cine neoleonés.

Hasta hace unos pocos años no se hablaba de una cinematografía que pudiera identificarse con la cultura neoleonesa; sin embargo, el estreno y éxito de películas como Inspiración (Ángel Mario Huerta, 2001), Las lloronas (Lorena Villarreal, 2004), Así (Jesús Mario Lozano, 2005), 7 días (Fernando Kalife, 2006) y Cumbia callera (René Villarreal, 2007) han puesto en la escena nacional e internacional las producciones de una generación de cineastas locales que están impulsando la cinematografía mexicana con sus propuestas fílmicas. El 20\% de la producción cinematográfica nacional en el 2003 y en el 2006 fueron películas realizadas en Nuevo León. De los 60 largometrajes producidos en el 2006, 13 fueron realizados por cineastas neoleoneses. Algunas de estas películas recibieron reconocimientos y premios, situando a las producciones locales a la par que las mejores nacionales (Hinojosa, 2007b, p. 15).

Estas nuevas generaciones de cineastas aseguran una cierta permanencia de las producciones cinematográficas de Nuevo León en las salas nacionales e internacionales, ya que con su actividad han generado nuevas alternativas de producción y distribución, sin dejar de lado una propuesta cinematográfica regional. 
En una entrevista realizada al cineasta regiomontano Jesús Mario Lozano, y publicada por Paul Julian Smith (2014), a la pregunta de cómo veía la relación entre el regionalismo y el centralismo de Ciudad de México en el mundo del cine mexicano, el cineasta respondió:

Eisenstein escribió en sus memorias que Monterrey era un lugar horrible en el cual no había nada qué hacer y estuvo en lo correcto de alguna manera. Por largo tiempo he tratado de producir películas aquí sin tener que recurrir a la capital del país, no tanto porque ame la ciudad de Monterrey, sino porque creo que hay 'otros cines' en el país, que existen y son aplastados por una hegemonía centralista que realmente sofocan. Al mismo tiempo he querido hacer del cine del lugar de donde provengo, una especie de 'geo-cinema', que responda al clima caliente, a las montañas, a la aridez (Citado en Smith, 2014, p. 230).

Esta especie de "geo-cinema", al que alude Jesús Mario Lozano en la entrevista, puede interpretarse en términos de territorio: desterritorializar y reterritorializar la producción cinematográfica, descentralizarla, que las diferentes idiosincrasias que existen en el país, la multiculturalidad de sus regiones, la particularidad de sus cineastas y su entorno geográfico y cultural, tengan cabida en la cinematografía nacional, una producción cinematográfica con su propio movimiento, un nuevo modo de producción con una identidad que la distinga de las demás, enraizada en su territorio de origen, sus circunstancias socio-históricas, sus valores culturales, su conciencia del mundo, donde dentro de cada película, como un microcosmos, se reterritorialicen también la palabra y la imagen.

\section{De lo local a lo global: EN BUSCA DE UN CINE "REGIO"}

Con el propósito de ahondar acerca de si se puede hablar de un cine que se identifique en la actualidad como neoleonés, se llevaron a cabo una serie de entrevistas a tres programadores y organizadores de festivales de cine en Monterrey y a tres cineastas locales. Del grupo de organizadores y programadores de festivales de cine, se entrevistó a: Carlos García Campillo, coordinador de Programación y Acervo de la Cineteca Nuevo León; Juan Manuel González, director del Festival de Cine en Monterrey; y Narce Dalia Ruiz, programadora del mismo festival. Entre los cineastas entrevistados se encuentran Fernando Kalife ( 7 días, 2005; 180 , 2011 ;

Llegando a casa: Rayados de Monterrey, 2016; 108 costuras, 2018), Juan Carlos Salas (Paraguay 76, 2015; La leyenda del Señor de la Expiración, 2017) y Cecilia Serna (Vivos los llevaron, vivos los queremos, 2007) Las entrevistas se realizaron entre agosto y diciembre de 2018 en sus oficinas particulares en la ciudad de Monterrey, Nuevo León.

Entre las preguntas que se les hicieron fueron: ¿cuáles han sido las tendencias temáticas en la producción fílmica actual de la entidad?, ¿existen elementos de identidad neolonesa que podamos identificar en las películas realizadas por cineastas locales?, y ¿cuáles son los retos y dificultades a los que se enfrentan los cineastas locales?

En referencia a la primera pregunta, los organizadores coinciden en que no se ha consolidado una tendencia temática, ya que los cineastas han apostado más bien a la diversidad:

La verdad es que hay mucha variedad. Obviamente de las cuestiones sociales salen algunas cosas, cuestiones sobre todo alrededor de la violencia, que todos conocemos. Pero realmente no hay una tendencia. Los creadores de Nuevo León son muy diversos; y así como ellos son diversos, hay diversidad en sus temáticas (Carlos García Campillo, comunicación personal, 22 de marzo de 2018).

No considero que haya una tendencia en particular, creo que los cineastas están explorando temas, géneros, o posibilidades estilísticas (...) Habrá anclajes donde se habla del noreste, o donde se hable de Monterrey, pero son casi inevitables, porque es donde se está haciendo la película (Juan Manuel González, comunicación personal, 1 de octubre de 2018). 
Cuando Nuevo León tuvo los graves problemas de inseguridad, por el 2010, era muy claro que esa era la temática dominante. El resto del tiempo es un cine más de autor. [En el FIC Monterrey] hemos programado documentales sobre los matachines ${ }^{2}$ o sobre los desplazamientos de personas de otros estados hacia Nuevo León, o de Celso Piña; es decir, temáticas muy de la región, pero sin que domine un tema (Narce Dalia Ruiz, comunicación personal, 4 de octubre de 2018).

Por su parte, los cineastas coinciden en que sus películas buscan ser motivadoras, dejar un mensaje o una reflexión. Solo uno de ellos se refirió a temas específicos tratados en sus filmes.

Me gusta tratar historias de cambio; las mejores historias siempre deben de hablar de un tipo de arco o algún tipo de hipérbole del carácter. Pero me gustan las historias que hablan acerca de la transformación de una mujer, un hombre, un niño, o un adulto; el perdón, la reconciliación, la visión, la amplitud, o el crecimiento, son temas que los encuentras en $\mathbf{7}$ días 0 en $\mathbf{1 8 0}^{\circ}$; el deporte y el futbol también son temas recurrentes en mis películas (Fernando Kalife, comunicación personal, 14 de septiembre de 2018).

Por supuesto, la tendencia temática para mí es que las historias tienen que ser inspiracionales y tienen que aportar algún valor propositivo (Juan Carlos Salas, comunicación personal, 17 de septiembre de 2018).

Me interesan las historias que dejen un mensaje, o bien una reflexión de la vida, la sociedad y la espiritualidad (Cecilia Serna, comunicación personal, 4 de octubre de 2018).

Acerca de la segunda pregunta, sobre si se pueden reconocer elementos que identifiquen una identidad neoleonesa en películas realizadas por cineastas locales, los organizadores no llegaron a un punto de acuerdo:

${ }^{2}$ Los matachines son grupos de danzantes de todas las edades, ataviados con penachos y trajes típicos de la región, que acompañan a las tradicionales peregrinaciones de la religión católica para rendir culto a la Virgen de Guadalupe en su Basílica cada año, desde mediados de octubre hasta el 12 de diciembre.
De hecho, ese es un problema del cine hecho en Nuevo León, que precisamente no hay esos identificadores. Con creadores que ya tienen un poquito más de carrera como guionistas o productores, como René Villarreal o Jesús Mario Lozano, se empiezan a notar temas como la desigualdad de clases; pero en cinematografias más incipientes o inmaduras lo que hay son, por ponerle una palabra, clichés, como el fútbol, la cerveza, la carne asada... (Carlos García Campillo, comunicación personal, 22 de marzo de 2018).

La realidad es que hay veces que los cineastas intentan esconder el origen de la película (...) pero en otros casos muestran la ciudad. Por ejemplo,

Cumbia callera está filmada en la colonia Independencia, en el centro de Monterrey. Se ve el Cerro de la Silla, se ven panorámicas de la ciudad (...) Hay otras películas donde son selectivos; por ejemplo, en el caso de Inspiración, de Ángel Mario Huerta, en que se muestra San Pedro Garza García, pero no se muestra Monterrey, por lo que hay una selectividad en cómo se muestra el entorno. Aparte de eso, sí hay señales de una identidad, más regional que regiomontana. Lo que te decía, de una cercanía con el desierto. Evidentemente aparecerán cuestiones que tienen que ver con la comida, la carne asada, cosas que son muy típicas de la región, que ya habría que hacer un análisis más detallado de en qué películas aparece tal cosa, pero no me parece que sea necesariamente una preocupación particular de los cineastas (Juan Manuel González, comunicación personal, 1 de octubre de 2018).

Personalmente no identifico una identidad, porque las lineas son muy variadas. Hay temáticas muy neoleonesas, como lo de Celso Piña, o los documentales Flores para el soldado $o$ El hombre de las gerberas, que hablan de situaciones que pasan en la colonia Independencia. Pero de forma global, en todas las películas que hemos seleccionado en los últimos 10 años, sobre todo en las ficciones, yo no encontraría una línea de identidad, ni de clase social, ni cultural; es muy variopinta (Narce Dalia Ruiz, comunicación personal, 4 de octubre de 2018).

Por su parte, los cineastas mencionan ser conscientes de elementos distintivos de la identidad neoleonesa en sus películas. Dos de ellos lo explican con detalle. 
La trama de [mi primera película], 7 días, se trataba de un joven empresario que quería traer un gran evento a su ciudad de Monterrey; y en $\mathbf{1 8 0}^{\circ}$, aunque filmado aquí, lo hicimos un poquito más universal, con un sentimiento que pudiera entenderse en prácticamente cualquier parte del país, aunque con escenarios nuestros; la última ficción que dirigí,

108 costuras, la cual es de béisbol, que es importante en la ciudad (Fernando Kalife, comunicación personal, 14 de septiembre de 2018).

Todo el tiempo me gusta incluir cosas de mi región. Por ejemplo, en mi primer cortometraje inclui lo que era la alimentación, la cercanía que hay en la familia, o la forma en que se celebra la Navidad; quería compartirlo al resto del mundo. Mi último cortometraje, La leyenda del Señor de la Expiración, es sobre las raíces de los pueblos tlaxcaltecas, los primeros pobladores de Nuevo León (Juan Carlos Salas, comunicación personal, 17 de septiembre de 2018).

Buscando ahondar en la cuestión de la identidad, a los cineastas se les preguntó si para ellos existe el concepto de "cine regiomontano" como tal, es decir, un cine que por sus características sea reconocido como "hecho en Nuevo León". Dos de los entrevistados respondieron que tal vez lo hubo en una época, señalando en particular los años en que se filmaba el llamado cabrito western, pero que en la actualidad no puede llamársele así al cine que se realiza en la entidad; sin embargo, una tercera entrevistada señala que sí existe, enfocándose más en los escritores, directores y productores del estado que en las características de los filmes.

Creo [que existió] en alguna época, la de todos los pioneros, que con una mano en la cámara y la otra tratando de conseguir recursos, hubo más ese feeling, ese sentimiento; luego hubo empresarios importantes que hicieron peliculas en los 80 y que lograron mantenerse en cartelera 6, 10 o hasta 12 meses. Por aquel tiempo hubo un cine que de alguna u otra forma era el cine regiomontano (...) Luego no se hizo nada por décadas y después hemos hecho unas películas en la región que tienen un toque de origen regiomontano, pero no lo veo tan actual (Fernando Kalife, comunicación personal, 14 de septiembre de 2018).
No lo creo, no creo que haya un cine que digas 'este es de Nuevo León'. En su tiempo hubo lo del cabrito western, que eso sí identificaba [con la región], porque era estilo muy norteño; pero no, definitivamente no creo que haya algo que lo distinga ahora (Juan Carlos Salas, comunicación personal, 17 de septiembre de 2018).

Podría decirse que sí, pues en la actualidad cada vez son más los escritores, directores y productores en el estado de Nuevo León (Cecilia Serna, comunicación personal, 4 de octubre de 2018).

Finalmente, en cuanto a los retos y dificultades a las que se enfrentan los cineastas al realizar sus películas, los tres programadores y organizadores coinciden en que son los mismos a los que se enfrentan todos los cineastas del mundo:

Los problemas son los mismos en todo el país, y me atrevería a decir en la mayoría de países del mundo. Es el acceso a financiamiento, para hacer producciones más profesionales; mano de obra especializada, que existan perfiles de profesionales, que sepan hacer lo que les toca hacer; y ventanas de distribución de las películas ya hechas. Esas son las principales dificultades (Narce Dalia Ruiz, comunicación personal, 4 de octubre de 2018).

Los cineastas coinciden en lo señalado por los organizadores, sin embargo, añaden un elemento sui generis de su condición de artistas, y es el tener una buena historia que contar.

En realidad son muchísimos retos, pero creo que el primero es poder tener una historia sólida, porque una cosa es tener la idea y otra tener una historia redonda que se pueda contar a través del cine (...) Muchos artistas no se preocupan por compartir o revisar previamente [su guion], y simplemente escriben lo que ellos piensan; lo realizan, ejecutan y eso es más como cine de autor. Otros tantos nos preocupamos porque tenga una estructura dramática en la cual se pueda entender la historia; y pensamos mucho antes de hacer los rodajes (Juan Carlos Salas, comunicación personal, 17 de septiembre de 2018). 


\section{Conclusiones}

Lo que presentamos en este artículo es solo una aproximación al cine hecho en Nuevo León. Sin embargo, esta primera exploración nos devela algo de la condición del cine hecho en la entidad, el cual está vinculado a la propia situación de la cinematografía nacional.

La historia reciente del cine mexicano nos muestra que, luego de una etapa de gran crisis, este se recupera, mostrando incrementos en la producción, número de salas de cine, indicadores de asistencia y taquilla; una transformación en su nueva etapa y la vuelta del público que tiene un gran papel en su recuperación; pero seguimos viendo una continuidad en la escasa exhibición en salas y en la inercia de nuestros legisladores para no trabajar en una reforma legislativa del sector que propicie las condiciones estructurales para que el cine mexicano tenga un fomento, promoción y difusión sostenidos. Estas mismas determinaciones viven los cineastas de Nuevo León, aunado a la centralización que existe en la producción.

A través de temáticas diversas, algunas de ellas ancladas al paisaje territorial, la cinematografía neolonesa explora géneros y estilísticas vinculados a ese concepto de "geo-cinema", señalado por Jesús Mario Lozano: desterritorializar para reterritorializar el cine, tratando de mostrar un cine que, por sus características artísticas y modo de hacer filmico, se distinga y encuentre un lugar propio en el mundo cinematográfico.

En este reterritorialización, y a través de su exploración de géneros y estilos, algunos de estos filmes rescatan valores locales como el de la familia, las celebraciones religiosas y prácticas culturales como el deporte; otros despliegan aspectos más controversiales como la desigualdad de clases, la discriminación, la violencia y la delincuencia; o intimistas, como la soledad y los conflictos personales, destacando aspectos escenográficos que propician identificar elementos regionales como el Cerro de la Silla y la colonia Independencia (Cumbia callera, de René Villarreal), el municipio de San Pedro Garza García (Inspiración, de Ángel Mario Huerta), o la avenida Constitución junto al río Santa Catarina ( 7 días, de Fernando Kalife).

En una última reflexión y ante la pregunta de si existe el concepto de "cine regiomontano" como tal, es decir, un cine que por sus características sea reconocido como "hecho en Nuevo León", coincidimos con quienes señalaron que tal vez así se podría identificar a la producción de una época pasada, aludiendo al cabrito western y a las películas de bandoleros, por considerarlo "muy norteño". Sin embargo, hoy en día son más los cineastas que producen en la entidad, que pertenecen a una nueva generación y tienen una formación universitaria, y que su producción es pensada para ser distribuida no solo de manera tradicional, sino considerando múltiples plataformas de exhibición. Sus temáticas trascienden, o entrecruzan, los límites entre lo local y lo global, lo que le confiere a su cinematografía una gran diversidad y alcance para públicos cada vez más heterogéneos y exigentes.

Como vemos, el cine neoleonés está en busca de un lugar en la cinematografía nacional e internacional, y si bien todavía se padece un centralismo en cuanto a la producción, es de destacarse la perseverancia con la que los cineastas neoloneses tratan de colocar sus producciones en el mapa del mercado cinematográfico mundial. 


\section{Bibliografía}

Cámara Nacional de la Industria Cinematográfica. (2019). Resultados definitivos 2018. Recuperado de: http://canacine.org.mx/wp-content/uploads/2019/02/ Resultados-definitivos $\% \mathrm{C} 2 \% \mathrm{~B} 418-6 . p d f$

Consejo Nacional de Población. (s. f.). Indicadores demográficos de México de 1950 a 2050. Recuperado de http://www.CONAPO.gob.mx/work/models/CONAPO/ Mapa_Ind_Dem18/index_2.html

Filmografia Mexicana. (s. f.). Ciudad de México, México: Filmoteca UNAM. Recuperado de http://www.filmografiamexicana.UNAM.mx/index.html

GonzÁlez, D. y Maza, M. (2013). Nuevo León en el cine. Monterrey, México: COnARTe, CONACULTA.

Hinojosa, L. (2003). El cine mexicano: de lo global a lo local. Ciudad de México, México: Trillas.

Hinojosa, L. (2007a). El cine mexicano: la identidad culturaly nacional. Ciudad de México, México: Trillas.

Hinojosa, L. (2007b). Una historia local en el horizonte mundial: el cine mexicano en Monterrey. Global Media Journal México, 4(8), 1-23. Recuperado de https://journals. tdl.org/gmjei/index.php/GMJ_EI/article/view/27

Hinojosa, L. (2019). Nueva época de oro para el cine mexicano: una mirada local en el horizonte mundial del mercado cinematográfico. Palabra clave, 22(3), e2235. doi: https://doi.org/10.5294/pacla.2019.22.3.5

Instituto Mexicano de Ginematografía. (2014). Anuario estadístico de cine mexicano 2013. Ciudad de México, México: Conaculta, imaine.

Instituto Mexicano de Ginematografía. (2015). Anuario estadístico de cine mexicano 2014. Ciudad de México, México: Gonaculta, imaine.

Instituto Mexicano de Ginematografía. (2016). Anuario estadístico de cine mexicano 2015. Ciudad de México, México: ConaCulta, imaine.

Instituto Mexicano de Ginematografía. (2017). Anuario estadístico de cine mexicano 2016. Ciudad de México, México: Conaculta, Imaine.

Instituto Mexicano de Ginematografía. (2018). Anuario estadístico de cine mexicano 2017. Ciudad de México, México: Conaculta, IMcine.

Instituto Mexicano de Ginematografía. (2019). Anuario estadístico de cine mexicano 2018. Ciudad de México, México: ConaGulta, imaine.

Instituto Mexicano de Cinematografía. (2020). Anuario estadístico de cine mexicano 2019. Ciudad de México, México: ConaGulta, imaine.

Sifuentes Zúñiga, K. D. (2019). Historia social del cine en Monterrey durante el Porfiriato y la Revolución Mexicana (1898-1927). Monterrey, México: Consejo para la Cultura y las Artes de Nuevo León.

Smith, P. J. (2014). Mexican Screen Fiction. Cambridge, Reino Unido: Polity Press. 


\section{Filmografía}

Villarreal, R. (Director \& Productor) \& Ramírez González, O. (Productor). (2007).

Cumbia callera. México: Cumbia Regia Films.

Lu aila Hinojosa-Ciór dova (México) es Doctora en Ciencias de la Comunicación Social por la Universidad de La Habana, Cuba. Profesora investigadora en la Universidad Autónoma de Nuevo León desde 1990. Miembro del Sistema Nacional de Investigadores del CONACYT, nivel II. Sus líneas de investigación son la economía política del cine mexicano, los estudios culturales y la comunicación pública de la ciencia.

Karen Jasso Vaquera (México) es egresada de la Licenciatura y Maestría en Ciencias de la Comunicación de la Universidad Autónoma de Nuevo León. Actualmente se desempeña como profesionista independiente. 